\title{
Mesenchymal Stem Cells Attenuate the Renal Tubular Epithelial Cells Epithelial Mesenchymal Transition by Restoring the Reciprocal Balance between Hepatocyte Growth Factor and Transforming Growth Factor- $\beta 1$
}

Junjun Wei, Li Tang, Shuwei Zhang, Liangliang Chen, Zhenhua Xie, Ren Yu, Honggang Qi, Jiangyong Lou and Guobin Weng*

Department of Renal Transplantation, Ningbo Urology and Nephrology Hospital, School of Medicine, Ningbo University, Zhejiang, China

${ }^{*}$ Corresponding author: Guobin Weng, Department of Renal Transplantation, The Affiliated Urology and Nephrology Hospital, School of Medicine, Ningbo University, 998 Qianhe Road, Ningbo, Zhejiang 315100, P.R. China, Tel: +86-15858470370; Fax: +86-574-55662738; E-mail: wengguobin2018@126.com

Received March 23, 2018; Accepted April 11, 2018; Published April 30, 2018

\section{Retraction Note:}

The article entitled "Mesenchymal Stem Cells Attenuate the Renal Tubular Epithelial Cells Epithelial Mesenchymal Transition by Restoring the Reciprocal Balance between Hepatocyte Growth Factor and Transforming Growth Factor- $\beta 1$ ” has been accepted for publication in the Journal of Stem Cell Research \& Therapy: Open Access considering the statements provided in the article as personal opinion of the author which was found not having any conflict or biasness towards anything. As the article was a perspective one, information provided by the author was considered as an opinion to be expressed through publication.

Publisher took decision to make the article online solely based on the reviewers suggestion which considered the article not but a personal opinion of the author. However, it is found that the author have some personal concerns and issues, therefore, being retracted from the journal. 